\title{
EMPREGO DE FIBRAS DE TRIGO E SOJA NA ELABORAÇÃO DE MORTADELAS
}

\author{
Mireila Behling ${ }^{1}$ \\ Liliane Marquardt ${ }^{2}$ \\ Ana Lúcia Becker Rohlfes ${ }^{3}$ \\ Nádia de Monte Baccar ${ }^{4}$ \\ Mari Silvia R. de Oliveira ${ }^{5}$
}

\begin{abstract}
RESUMO
A mortadela está entre os produtos cárneos mais consumidos no Brasil. A substituição parcial de gordura animal por fibras vegetais na elaboração de mortadelas foi o objetivo do presente estudo, uma vez que as fibras possuem valor nutricional de interesse para a saúde humana, além de possuírem baixo custo. A gordura, por sua vez, é importante na formulação, pois atua na formação da emulsão. No desenvolvimento da pesquisa foram utilizadas três formulações de mortadela, sendo elas respectivamente: padrão (A), com substituição parcial de gordura por fibra de trigo (B) e com substituição parcial de gordura por fibra de soja (C). O estudo buscou atender o Regulamento Técnico de Identidade e Qualidade de Mortadela do Ministério da Agricultura, Pecuária e Abastecimento (MAPA), e as mortadelas foram avaliadas físicoquímicamente. Os resultados médios obtidos nas análises foram comparados aos citados pelo MAPA, sendo, respectivamente, para os produtos A, B e C: $60,4 \%, 59,3 \%$ e $61,1 \%$ de umidade; $5,5 \%, 5,3 \%$ e $4,5 \%$ de cinzas; $15,5 \%, 14,1 \%$ e $13,5 \%$ de gordura; $1,52 \%, 3,5 \%$ e $4,2 \%$ de fibras e 16,32\%, 17,2\% e 15,9\% de proteína. Estes resultados demonstram a viabilidade tecnológica para elaboração de novos produtos cárneos.
\end{abstract}

Palavras-chave: Mortadela. Gordura animal. Fibras vegetais.

\section{ABSTRACT}

The mortadella is among the most consumed meat products in Brazil. The partial replacement of animal fat for vegetable fibers in preparing mortadella was the purpose of this study, since the fibers have a higher nutritional value for human health, besides having a low cost. The fat is important in the preparing of mortadella, as it acts in the formation of the emulsion. In the study were used three formulations for the mortadella, which were respectively: standard (A), with partial fat replacement by wheat fiber, (B) partial fat replacement by soy fiber (C). The study sought to meet the Technical Rules of Identity and Quality of Mortadella by the Ministry of Agriculture, Livestock and Supply (MAPA) and the mortadella was evaluated physico-chemically. The average results obtained in this study were comparable to those cited by MAPA, being respectively to the mortadella A, B and C: $60,4 \%, 59.3 \%$ and $61.1 \%$ of

\footnotetext{
1 Acadêmica do Curso de Química Licenciatura da Universidade de Santa Cruz do Sul - UNISC. <mireilabehling@hotmail.com>

${ }^{2}$ Professora do Departamento de Engenharia, Arquitetura e Ciências Agrárias da Universidade de Santa Cruz do Sul - UNISC. <liliane@unisc.br>

${ }^{3}$ Professora do Departamento de Química e Física da Universidade de Santa Cruz do Sul - UNISC. <albecker@unisc.br>

${ }^{4}$ Professora do Departamento de Química e Física da Universidade de Santa Cruz do Sul - UNISC. <nadia@unisc.br>

5 Professora do Departamento de Química e Física da Universidade de Santa Cruz do Sul - UNISC. <mari@unisc.br>
} 
moisture, $5.5 \%, 5.3 \%$ and $4.5 \%$ of ash, $15.5 \%, 14.1 \%$ and $13.5 \%$ of fat; $152 \%, 3.5 \%$ and $4.2 \%$ of fiber, and $16.32 \%, 17.2 \%$ and $15.9 \%$ of protein. These results demonstrate the technological feasibility for developing new meat products.

Keywords: Mortadella. Animal fat. Vegetable fibers.

\section{INTRODUÇÃO}

A população está cada vez mais preocupada com sua qualidade de vida e, muitas vezes, essa preocupação leva à transformação de hábitos e costumes, principalmente, no que diz respeito à alimentação. Percebendo esse fato, o mercado alimentício busca promover mudanças e melhorias em seus produtos, agradando, assim, as expectativas do consumidor, que está cada vez mais exigente. Esta tendência levou à criação de uma nova área de investigação nas ciências dos alimentos e da nutrição: os alimentos funcionais (DEVITTE; DINON, 2011).

Segundo os mesmos autores, a mortadela, cujo consumo no Brasil se popularizou ao longo dos anos, é um produto cárneo industrializado com potencial de tornar-se um alimento funcional, se houver a possibilidade de adicionar fibras vegetais nas formulações. Conhecida pela cor rosa, sabor delicado de massa fina, aroma suave e como ingrediente de lanches, a mortadela apresenta grande procura entre os itens alimentícios. Segundo estimativas de analistas do setor de alimentos, a produção apresenta uma média que ultrapassa 100 mil toneladas anuais no País.

A inclusão de fibras na dieta humana está relacionada com o bem-estar e a boa saúde dos indivíduos. A adequada ingestão previne ou ameniza problemas como constipação, obesidade, câncer de colo-retal, diverticulite, diabetes e doenças cardiovasculares associadas a elevados teores sanguíneos de colesterol e triglicerídeos (BARTOLOMEU, 2011).

Já, a diminuição do teor de gordura em mortadelas implica na redução de atributos sensoriais e de qualidade como maciez, suculência, textura, sabor, aparência e rendimento (KEETON, 1994; HUFFMAN; HUFFMAN, 1997). Segundo Jimenez-Colmenero (1996), com a redução do teor de gordura, é necessária a utilização de ingredientes não cárneos que permitam desenvolver uma combinação de outros ingredientes substituir tecnologicamente a gordura, e mantendo a emulsão estável (GUIMARAES, 2012).

Para Cáceres et al. (2004), várias fibras alimentares são utilizadas em produtos cárneos, não somente pelo efeito benéfico à saúde, mas também como potencial substituto de gordura. Já, segundo Keeton (1994) e Cyrino \& Barretto (2006), as fibras são empregadas por 
terem odor neutro, e apresentarem boa capacidade de reter a água, com grande benefício à saúde, baixo valor calórico, além de melhorarem o fatiamento de produtos.

\section{REVISÃO DE LITERATURA}

\subsection{Produtos cárneos}

Segundo Terra (1998), produtos cárneos são aqueles cuja matéria-prima principal e que dá característica ao produto, é a carne. Podem ser processados, principalmente, a partir de carne bovina, suína e de frango. São, de preferência, obtidos a partir de carne fresca que sofra um ou mais tipos de processo, entre eles, cozimento, salga, defumação ou mesmo somente a adição de condimentos e temperos (BENEVIDES; NASSU, 2010). Entende-se por embutidos, os produtos constituídos à base de carne picada e condimentada, com forma geralmente simétrica e, que são embutidos sob pressão em um recipiente ou envoltório de origem orgânica ou inorgânica, aprovado para este fim (TERRA, 1998).

Uma emulsão pode ser definida como sendo uma suspensão coloidal de dois líquidos imiscíveis, mas que, no entanto, mantêm-se dispersos um no outro, pela ação de um agente emulsificante interfacial, que, em alimentos, é a proteína. Quando a carne, gordura, água e sal são misturados e submetidos à alta velocidade de cominuição, uma massa homogênea é formada, com características de emulsão, visto que os três componentes básicos para formá-la estão presentes (água, gordura e proteína) (SHIMOKOMAKI et al., 2006).

A proteína cárnea, especialmente a miofibrilar, por possuir uma porção hidrofóbica (apolar) e outra hidrofílica (polar), atua na interface entre a gordura e a água, permitindo a formação da emulsão (PARDI et al., 1993).

\subsection{Mortadela}

A mortadela, por sua vez, pode ser definida, como um produto cárneo industrializado, obtido de uma emulsão de carnes animais de açougue, acrescido ou não de toucinho, adicionado de ingredientes, embutidos em envoltório natural ou artificial, em diferentes formas, e submetido ao tratamento térmico adequado (MAPA, 2000). Quanto às características sensoriais, a mortadela deverá apresentar textura, cor, sabor e odor característicos.

Segundo o Regulamento técnico de identidade e qualidade de carne mecanicamente separada de mortadela, de linguiça e de salsicha, o teor de carboidratos totais (máximo) deverá ser de 1 a 10\%. E em mortadelas Bologna e Italiana (máximo), 3,0\%. Ainda, quanto ao amido (máximo), deve ser de 1 a 5,0\% e a umidade (máximo) 65\%. Já, o valor máximo 
estabelecido para o teor de gordura é de $30 \%$, sendo que, para mortadelas Bologna e Italiana, é, no máximo, de 35\%. (MAPA, 2000).

\subsection{Fibras}

No Brasil, segundo a Agência Nacional de Vigilância Sanitária (ANVISA), em sua Resolução RDC n. 360, de 23/12/2003 (BRASIL, 2003), a fibra alimentar é definida como qualquer material comestível, consumido normalmente como componente de um alimento que não seja hidrolisado pelas enzimas endógenas do trato digestivo humano.

Saad (2006) define a fibra alimentar como sendo o componente da parede celular dos vegetais incluído na dieta humana que resiste à ação das secreções do trato gastrointestinal. Em termos bioquímicos, as fibras são um grupo de moléculas que formam um composto químico complexo e dividem-se em duas categorias: insolúveis e solúveis. As insolúveis, encontradas em cereais (farelos), hortaliças, frutas (com cascas) e leguminosas, atuam principalmente na parte inferior do intestino grosso, ampliando o bolo fecal. Já as solúveis, disponíveis na aveia, na cevada, no bagaço de frutas cítricas, na maçã e na goiaba, por exemplo, agem no estômago e no intestino delgado, fazendo com que a digestão seja mais lenta. A fibra é adequada para uso em produtos cárneos, em especial, nas emulsões cárneas, porque retém água, diminui perda pelo cozimento e possui sabor neutro (MARTINS, 1997).

O objetivo foi avaliar a possibilidade técnica de substituir parcialmente a gordura animal por diferenciadas fibras vegetal, como fibra de trigo e fibra de soja.

\section{METODOLOGIA}

\subsection{Elaboração da mortadela}

A partir de estudos teóricos referentes ao processamento de mortadelas e ao benefício do emprego de fibras vegetais na alimentação humana, iniciou-se a parte experimental de elaboração do produto emulsificado - mortadela com a substituição parcial de gordura por fibra vegetal.

A metodologia para o processamento de mortadelas foi desenvolvida na Planta Piloto de Produtos de Origem Animal, da Universidade de Santa Cruz do Sul - RS, e baseou-se em Terra (1988), cujo procedimento consta no fluxograma da Figura 1. 
Figura 1 - Fluxograma de metodologia da formulação de mortadela.

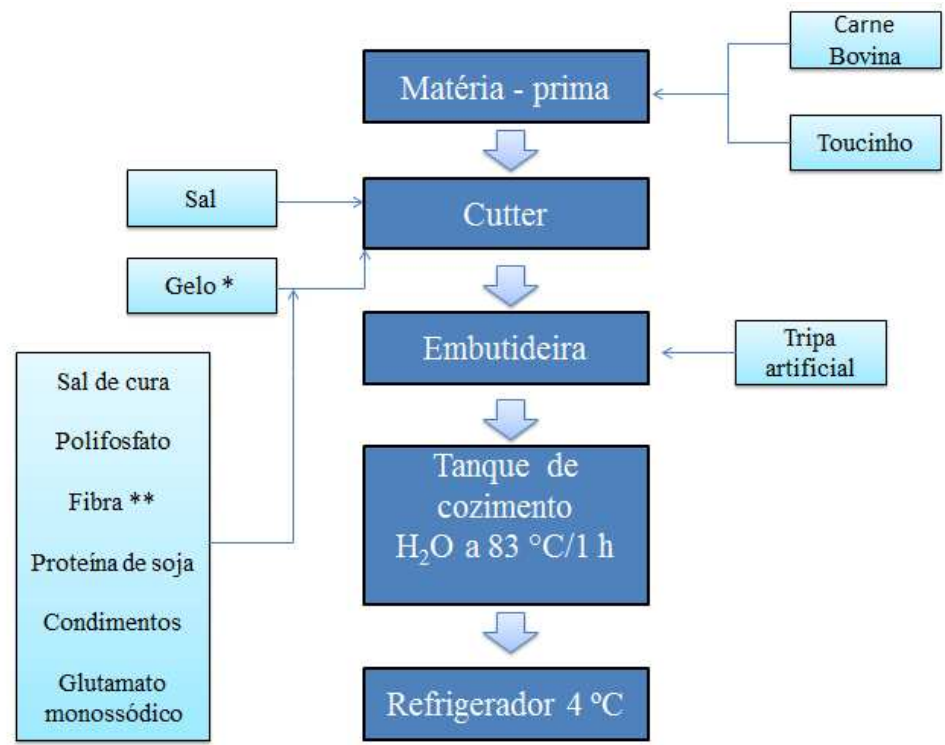

*sempre intercalando com os demais ingredientes.

**apenas para formulação de substituição.

Na elaboração de mortadelas com a substituição parcial de gordura (toucinho) por fibra de trigo e soja, estas foram acrescentadas após a adição de sal de cura. Os produtos elaborados foram codificados como: mortadela sem adição de fibra vegetal (A), mortadela com adição de fibra de trigo (B) e mortadela com adição de fibra de soja (C). As formulações desenvolvidas constam na Tabela 1.

Tabela 1 - Formulação das mortadelas A, B e C.

\begin{tabular}{lccc}
\hline \multicolumn{1}{c}{ Matéria - prima } & $\begin{array}{c}\text { Mortadela A } \\
(\mathrm{g})\end{array}$ & $\begin{array}{c}\text { Mortadela B } \\
(\mathrm{g})\end{array}$ & $\begin{array}{c}\text { Mortadela C } \\
(\mathrm{g})\end{array}$ \\
\hline Carne bovina & 1000,0 & 1000,0 & 1000,0 \\
Toucinho & 333,0 & 323,0 & 323,0 \\
Fibra & - & 10,0 & 10,0 \\
Sal & 25,0 & 25,0 & 25,0 \\
Polifosfato de sódio & 66,0 & 66,0 & 66,0 \\
Gelo & 386,0 & 386,0 & 386,0 \\
Proteína concentrada de soja & 26,0 & 26,0 & 26,0 \\
Condimento de sabor mortadela & 13,0 & 13,0 & 13,0 \\
Sal de cura & 3,2 & 3,2 & 3,2 \\
Glutamato monossódico & 3,2 & 3,2 & 3,2 \\
Alho & 2,6 & 2,6 & 2,6 \\
Pimenta preta moída & 1,3 & 1,3 & 1,3 \\
\hline
\end{tabular}

\subsection{Avaliação da composição centesimal}

As amostras de mortadelas A, B e C foram avaliadas quanto à composição centesimal segundo a metodologia descrita por Cecchi (2003) e os procedimentos 
experimentais foram realizados no laboratório de bromatologia do curso de química industrial da Universidade de Santa Cruz do Sul - RS.

\subsection{Análise estatística}

A análise estatística foi realizada com o emprego do software STATISTC ${ }^{@}$ 10.0, utilizando a análise de variância (ANOVA) com $5 \%$ de probabilidade pelo teste F.

\section{RESULTADOS}

\subsection{Processamento de mortadelas}

A Figura 2 apresenta o aspecto das massas de mortadelas A, B e C antes da etapa de embutimento e cozimento.

\section{Figura 2 - Massa de mortadelas A, B e C.}

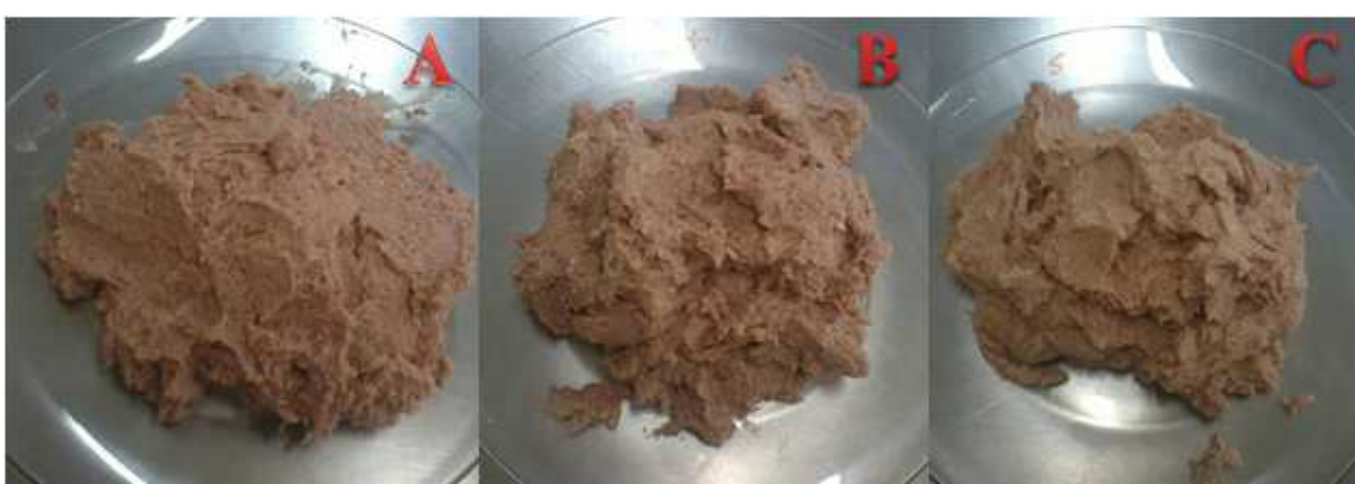

Visualmente percebe-se diferença na coloração e na consistência das massas e, de acordo com Dias (2007), a alteração na consistência deve-se, principalmente, à propriedade tecnológica das fibras vegetais empregadas em formulações de produtos cárneos de absorver a água que foi adicionada para facilitar a formação da emulsão entre a proteína miofibrilar da carne e a gordura animal.

\subsection{Análises físico-químicas}

Os resultados das análises físico-químicas de umidade, lipídeos, cinza, proteínas e fibras, realizadas com amostras das três formulações de mortadelas (A, B e C) encontram-se na Tabela 2. 
Tabela 2 - Composição centesimal das mortadelas A, B e C.

\begin{tabular}{cccc}
\hline Análise & Mortadela A $(\%)$ & Mortadela B $(\%)$ & Mortadela C (\%) \\
\hline Umidade & $60,49^{\mathrm{a}} \pm 0,16$ & $59,31^{\mathrm{b}} \pm 0,32$ & $61,18^{\mathrm{a}} \pm 0,06$ \\
Lipídeos & $15,59^{\mathrm{a}} \pm 0,50$ & $14,10^{\mathrm{b}} \pm 0,14$ & $14,51^{\mathrm{b}} \pm 0,15$ \\
Cinzas & $5,56^{\mathrm{a}} \pm 0,10$ & $5,35^{\mathrm{a}} \pm 0,53$ & $4,55^{\mathrm{a}} \pm 0,01$ \\
Proteínas & $16,34^{\mathrm{a}} \pm 0,98$ & $17,25^{\mathrm{b}} \pm 0,42$ & $15,91^{\mathrm{a}} \pm 0,19$ \\
Fibras & $1,52^{\mathrm{a}} \pm 0,04$ & $3,51^{\mathrm{b}} \pm 0,03$ & $4,23^{\mathrm{c}} \pm 0,02$ \\
\hline
\end{tabular}

O resultado da quantificação dos teores médios de umidade, lipídios, cinzas, proteínas e fibras, obtido nas análises realizadas com amostras das mortadelas A, B e C atendem ao permitido pela legislação (MAPA, 2000). A mortadela B apresentou diferença significativa no teor de proteínas quando comparada às amostras de mortadelas A e C. Este fato deve-se, provavelmente, ao percentual de proteínas presente na fibra de trigo. Ainda, ao analisar os dados da Tabela 2, percebe-se que o teor de umidade da amostra B é significativamente menor que as demais amostras. Estes dois fatores (percentual de proteínas e de umidade) demonstram a viabilidade técnica na substituição parcial de gordura por fibra de trigo, uma vez que o menor teor de umidade aumenta a vida de prateleira desta formulação.

Quanto aos teores de lipídios, as amostras B e C não apresentaram diferença significativa entre si. Porém, quando comparadas à amostra A, percebe-se redução do valor médio resultante, o que demonstra a possibilidade de redução do percentual de gordura na formulação de mortadelas, quando esta é substituída por fibras vegetais de trigo e soja, sem alteração na estabilidade da emulsão obtida. Resultados semelhantes foram descritos por Guimarães (2011) em estudo realizado com produção de mortadelas com adição de fibras funcionais e redução de gordura.

\section{CONCLUSÃO}

O estudo desenvolvido com a elaboração de mortadelas a partir da substituição parcial de gordura por fibras de trigo e soja é viável tecnologicamente. Os resultados das análises físico-químicas demonstram que estes produtos estão de acordo com os padrões de identidade e qualidade de mortadelas e, que as características visuais (cor e textura) foram mantidas, independentemente do emprego de fibra de trigo ou de soja.

\section{REFERÊNCIAS}

BARTOLOMEU, D. A. F. S. Desenvolvimento e avaliação da aceitação de embutido defumado "tipo mortadela" elaborado com CMS de tilápia do Nilo (Oreochromis niloticus) $e$ 
fibra de trigo. 2011. 121 f. Dissertação (Programa de Pós-Graduação em Tecnologia de Alimentos) - Universidade Federal do Paraná, Curitiba, 2011.

BENEVIDES, S. D.; NASSU, R. Produtos cárneos. Brasília: Embrapa, 2010.

BRASIL. Agência Nacional de Vigilância Sanitária (ANVISA). Resolução RDC n³60: de 23 de dezembro de 2003. Regulamento Técnico sobre Rotulagem Nutricional de Alimentos Embalados. Disponível em:

<http://portal.anvisa.gov.br/wps/wcm/connect/1c2998004bc50d62a671ffbc0f9d5b29/RDC_N 360_DE_23_DE_DEZEMBRO_DE_2003.pdf?MOD=AJPERES> Acesso em: 28 de mar. de 2014.

CÁCERES, E.; GARCÍA, M. L.; TORO, J.; SELGAS, M. D. The effect of fructooligosaccharides on the sensory characteristics of cooked sausages. Meat Science, Barking, n. 1,v. 68, p. 87-96, 2004.

CECCHI, H. M. Fundamentos teóricos e práticos em análise de alimentos. Campinas: Ed. da UNICAMP, 2003.

CYRINO, N. A.; BARRETTO, A. C. S. O que a Vitacel pode fazer aos seus embutidos. Revista nacional da carne, São Paulo, n. 352, v. 1, p. 110-111, 2006.

DEVITTE, S.D.S.L.; DINON, S. Mortadela adicionada de fibras e com substituição parcial da gordura por carragena e pectina. 2011. $47 \mathrm{f}$. Monografia. (Curso Superior de Tecnologia em Alimentos) - Universidade Tecnológica Federal do Paraná - Medianeira, 2011.

DIAS, A. A. Substitutos de gordura aplicados em alimentos para fins especiais. Brasília: UFB, 2007.

GUIMARAES, C. F. Formulação e caracterização de mortadela com adição de fibras funcionais e redução de gordura. 2012.108 f. Dissertação (Programa de Pós-Graduação em Ciência e Tecnologia de Alimentos) - Universidade Federal de Viçosa, Minas Gerais, 2012.

HUFFMAN, D.L.; HUFFMAN, R.D. Production of low fat and rediced fat ground beef. In: Prodution and processing of healthy meat, poultry and fish products. Advances in Meat Research Series, n. 1, v. 11, p. 226-241, 1997.

JIMENEZ-COLMENERO, F. Technologies for developing low-fat meat products. Food Science \& Technology, Cambridge, n. 1, v. 7, p. 41-48, 1996.

KEETON, J. T. Low-fat meat products - technological problems with processing. Meat Science, Barking, n. 1, v. 36, p. 261-276, 1994.

MAPA. Instrução Normativa $\mathrm{N}^{\circ}$ 4. Regulamento técnico de identidade e qualidade de carne mecanicamente separada, de mortadela, de linguiça e de salsicha. Brasília: Ministério da Agricultura e do Abastecimento, 2000.

MARTINS, C. Fibras \& fatos: como as fibras podem ajudar na sua saúde. Curitiba: NutroClínica, 1997.

PARDI et. al. Ciência, higiene e tecnologia da carne. Goiânia: UFG, 1993. 
SAAD, S.M.I. Probióticos e prebióticos: o estado da arte. Revista Brasileira de Ciências Farmacêuticas. n. 1, v. 42, p. 1-16, $2006 .$.

SHIMOKOMAKI et al. Atualidades em ciência e tecnologia de carnes. São Paulo: Varela, 2006.

TERRA, Nelcindo N. Apontamentos de tecnologia de carnes. São Leopoldo: Ed. UNISINOS, 1998. 